\title{
Theories of Context Effects in Multi-alternative, Multi-attribute Choice
}

\author{
Jennifer S. Trueblood ${ }^{1}$ (jennifer.s.trueblood@,vanderbilt),
}

Department of Psychology, Vanderbilt University

${ }^{1}$ Correspondence may be addressed to Jennifer S. Trueblood, Department of Psychology, Vanderbilt University, Nashville TN 37235, USA; Email:

jennifer.s.trueblood@,vanderbilt.edu 


\begin{abstract}
Over the past several decades, researchers in psychology, neuroscience, marketing, and economics have been keen to understand context effects in multi-alternative, multi-attribute decision-making. These effects occur when choices among existing alternatives are altered by the addition of a new alternative to the choice set. The effects violate classic decision theories and have led to the development of computational and mathematical models that explain how the effects arise due to underlying cognitive and neural mechanisms. This paper reviews the class of dynamic models of these effects, comparing mechanisms across models. We observe that most models of context effects incorporate an attention mechanism, suggesting attention plays an important role in multi-alternative, multi-attribute decision-making. We conclude by discussing recent empirical studies of attention and context effects and hypothesize that changes in attention could be responsible for recently observed reversals in context effects.
\end{abstract}

Keywords: evidence accumulation, preferential choice, context effects, attention, relative comparison 
Many of life's decisions - whether it be shopping for cereal or buying a home - involve multiple options that have different features. A core question in psychology, economics, marketing, and neuroscience is how do people make choices in these complex situations? Importantly, research has shown that the context created by a set of options influences people's choices. Over the past several decades, there has been extensive empirical and theoretical work on understanding "context effects" in multi-alternative, multi-attribute choice. Most of this research has focused on three "core" context effects: the attraction (Huber, Payne, \& Puto, 1982), compromise (Simonson, 1989), and similarity (Tversky, 1972) effects. These effects are empirical demonstrations of how choices between two existing options can change with the introduction of a third, new alternative.

To illustrate the effects, imagine you are deciding between two hotels for an upcoming trip (see Figure 1a). One of the hotels, call this hotel X, is in a great location (e.g., in walking distance to the best restaurants, shops, and museums), but is expensive. Whereas the other hotel, call this one $\mathrm{Y}$, is in a worse location, but is cheaper. Thus, there is a tradeoff in convenience and price for X and Y. Further, suppose that you are conflicted about this tradeoff and equally likely to select either X or Y. Now, suppose you learn of a third hotel, call this one A, that is similar to hotel $\mathrm{X}$, but is in a slightly worse location and a little more expensive than $\mathrm{X}$. A is termed a "dominated decoy" because option $\mathrm{X}$ is superior on both the attributes of price and convenience. In this case, A makes $\mathrm{X}$ look better and you ultimately choose $\mathrm{X}$. This phenomenon is known as the attraction effect.

In the similarity effect, the third option is similar to one of the existing options and competes with that option. In this case, the dissimilar option is selected. For example, consider a hotel called $\mathrm{S}$ that is similar to $\mathrm{Y}$ on both convenience and price dimensions. A similarity effect 
occurs when $\mathrm{X}$ is selected out of the choice set $\{\mathrm{X}, \mathrm{Y}, \mathrm{S}\}$. In the compromise effect, the third option is an extreme option that makes one of the existing options appear as a compromise. For example, consider a hotel called $\mathrm{C}$ that is in the best location, but very expensive. In the choice set $\{\mathrm{X}, \mathrm{Y}, \mathrm{C}\}, \mathrm{X}$ appears like a compromise between $\mathrm{C}$ and $\mathrm{Y}$. The compromise effect occurs when $\mathrm{X}$ is selected over $\mathrm{Y}$. Note that the difference between the similarity and compromise effects is related to the distance between the third option, $\mathrm{S}$ or $\mathrm{C}$, and the existing options. In the similarity effect, the third alternative is very close to one of the existing alternatives. In the compromise effect, the third alternative is an extreme alternative, further away from the existing alternatives.

\section{Figure 1}

Panel a: Two-dimensional attribute space illustrating the attraction ( $\mathrm{A}$ in red), compromise (C in blue), and similarity ( $\mathrm{S}$ in green) effects where $\mathrm{X}$ is the target. The economy attribute is the inverse of price (i.e., lower on economy implies a higher price). The colored regions show standard locations for the third (decoy) alternative for the three effects. Panel b: Schematic of a canonical preference accumulation model with three accumulators and internal stopping rule governed by the decision threshold. In this example, accumulator X reaches the threshold first and thus would be the option chosen.
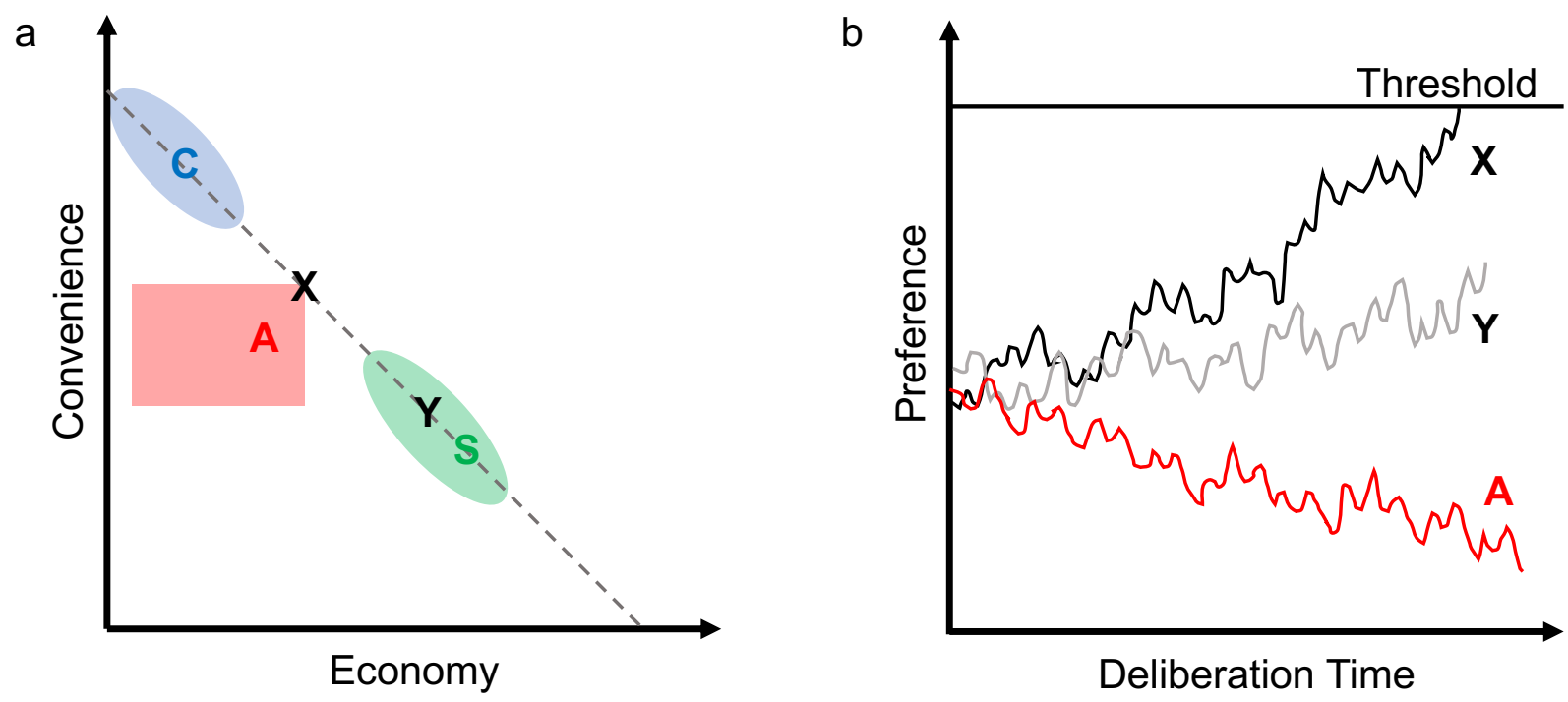
Context effects were originally used as examples of how people's choices violate the principles of classic decision theories. Luce's (1959) ratio of strengths model is an example of a theory violated by context effects. In this model, the probability of selecting an option is the strength $s$ of the option divided by the sum of the strengths of all options:

$$
p(X \mid\{X, Y, A\})=\frac{s(X)}{s(X)+s(Y)+s(A)} .
$$

Here the notation $\mathrm{p}(\mathrm{X} \mid\{\mathrm{X}, \mathrm{Y}, \mathrm{A}\})$ is the probability of choosing option $\mathrm{X}$ out of the choice set $\{\mathrm{X}, \mathrm{Y}, \mathrm{A}\}$. To see why the attraction effect leads to a violation of the model, consider two choice sets $\left\{\mathrm{X}, \mathrm{Y}, \mathrm{A}_{\mathrm{X}}\right\}$ and $\left\{\mathrm{X}, \mathrm{Y}, \mathrm{A}_{\mathrm{Y}}\right\}$ where $\mathrm{A}_{\mathrm{X}}$ and $\mathrm{A}_{\mathrm{Y}}$ are dominated decoys similar to $\mathrm{X}$ and $\mathrm{Y}$, respectively. The attraction effect implies $\mathrm{p}\left(\mathrm{X} \mid\left\{\mathrm{X}, \mathrm{Y}, \mathrm{Ax}_{\mathrm{X}}\right\}\right)>\mathrm{p}\left(\mathrm{X} \mid\left\{\mathrm{X}, \mathrm{Y}, \mathrm{A}_{\mathrm{Y}}\right\}\right)$ and according to the ratio of strengths model, we must have $\mathrm{s}\left(\mathrm{Ax}_{\mathrm{X}}\right)<\mathrm{s}\left(\mathrm{A}_{\mathrm{Y}}\right)$. On the other hand, the attraction effect also implies $\mathrm{p}\left(\mathrm{Y} \mid\left\{\mathrm{X}, \mathrm{Y}, \mathrm{A}_{\mathrm{X}}\right\}\right)<\mathrm{p}\left(\mathrm{Y} \mid\left\{\mathrm{X}, \mathrm{Y}, \mathrm{A}_{\mathrm{Y}}\right\}\right)$ and according to the ratio of strengths model, we must have $s\left(A_{X}\right)>s\left(A_{Y}\right)$. Since $s\left(A_{X}\right)<s\left(A_{Y}\right)$ and $s\left(A_{X}\right)>s\left(A_{Y}\right)$ cannot both be true, the assumptions of the model are violated. In particular, this is a violation of the assumption of "independence from irrelevant alternatives", which states that the probability of choosing an alternative over another does not depend on the choice set.

More recently, these effects have been instrumental in the development of formal models of multi-alternative, multi-attribute choice. Since they cannot be explained by classic decision theories, such the ratio of strengths model, theorists in psychology and neuroscience have been keen to understand the cognitive and neural mechanisms that give rise to the effects. Understanding the mechanisms that underlie context effects could shed light on how preferences form in multi-alternative, multi-attribute decision tasks more generally. 


\section{Dynamic Models of Context Effects}

For more than three decades, researchers have developed mathematical and computational models of context effects. At the time of writing, there are dozens of models of context effects across the fields of economics, marketing, psychology, and neuroscience. These models range from rational models based on Bayesian belief updating (Rigoli, Mathys, Friston, \& Dolan, 2017) to theories about normalization among populations of neurons (Dumbalska, Li, Tsetsos, \& Summerfield, 2020). In the current review, we focus on a subset of these models developed to explain the dynamics of multi-alternative, multi-attribute decision-making.

The class of models we consider here are known as evidence accumulation models (Ratcliff, 1978) and share some foundational assumptions. First, these models assume that during the course of deliberation, evidence (preference in the case of value-based decisions) for different options is accumulated. Each option is associated with an accumulator that tracks the evidence state for that option. Second, these models assume that decisions are determined by the amount of accumulated evidence for each option at either a fixed time (i.e., external stopping rule) or when the first accumulator reaches an internal decision threshold (i.e., internal stopping rule). In the latter case, evidence accumulation models account for both the choices people make and their response times (see Figure $1 \mathrm{~b}$ for an illustration). Evidence accumulation models are well suited to studying context effects because research has shown that these effects often change during the time course of deliberation (Pettibone, 2012; Cataldo \& Cohen, 2021), where the effects are small for quick decisions and larger for slow decisions.

To date, there are at least nine different dynamic models of context effects as listed in Table 1. Some of these models, such as Multi-alternative Decision Field Theory (MDFT; Roe, Busemeyer, \& Townsend, 2001), have been studied and tested extensively over the years. Other 
models, such as the Normalization and Urgency Model (Tajima, Drugowitsch, Patel, \& Pouget, 2019) and the Gaze-dependent Leaky Accumulator (GLA; Molter, Thomas, Huettel, Heekeren, $\&$ Mohr, 2021) are relatively new theories and thus have been studied less. Looking at Table 1, one might wonder which model is the "best". That is, which model provides the best account of context effects and multi-alternative, multi-attribute choice more generally. This turns out to be a difficult question to answer because while the models all have a similar foundation (i.e., being evidence accumulation models), they also have a number of additional assumptions that can make comparison difficult. Currently there have only been a handful of attempts to quantitatively compare some of these models, and those attempts have led to different conclusions (Noguchi \& Stewart, 2018; Turner, Schley, Muller, \& Tsetsos, 2018; Evans, Holmes, \& Trueblood, 2019). At the time of writing, there have been no attempts to compare all nine models in Table 1.

One promising avenue for future research on evaluating and comparing models is the “switchboard analysis" developed by Turner et al. (2018), where a model is viewed as a set of assumptions (e.g., how attention is processed, how attributes are compared, etc.) representing different decisions to be made by the modeler. In the switchboard approach, these decision points are referred to as "nodes" and the different options available at the decision points are referred to as "switches". A large family of possible models in generated by defining a set of switches for each possible node. Turner et al. (2018) used the switchboard approach to evaluate 432 different dynamic models of context effects. Their "nodes" and "switches" were defined using mechanisms from four of the models listed in Table 1 (i.e., MDFT, LCA, AAM, and MLBA). Thus, their approach provided a comprehensive comparison of all possible combinations of the mechanisms in these models. We think such an approach is very promising as it provides a thorough exploration of the space of possible models. 
Table 1

Dynamic Models of Context Effects and their Mechanisms

\begin{tabular}{|c|c|c|c|c|c|c|c|}
\hline Model Name & Reference & Attention & $\begin{array}{l}\text { Relative } \\
\text { Comparisons }\end{array}$ & Leakage & Inhibition & $\begin{array}{l}\text { Divisive } \\
\text { Normalization }\end{array}$ & Urgency \\
\hline $\begin{array}{l}\text { Multi-alternative } \\
\text { Decision Field } \\
\text { Theory (MDFT) }\end{array}$ & $\begin{array}{l}\text { Roe et al. } \\
(2001)\end{array}$ & $\mathrm{X}$ & $\mathrm{X}$ & $\mathrm{X}$ & $\mathrm{X}$ & & \\
\hline $\begin{array}{l}\text { Leaky Competing } \\
\text { Accumulator (LCA) }\end{array}$ & $\begin{array}{l}\text { Usher \& } \\
\text { McClelland } \\
\text { (2004) }\end{array}$ & $\mathrm{X}$ & $\mathrm{X}$ & $\mathrm{X}$ & $\mathrm{X}$ & & \\
\hline $2 \mathrm{~N}$-ary Choice Tree & $\begin{array}{l}\text { Wollschlager \& } \\
\text { Diederich } \\
\text { (2012) }\end{array}$ & $\mathrm{X}$ & $\mathrm{X}$ & $\mathrm{X}$ & $\mathrm{X}$ & & \\
\hline $\begin{array}{l}\text { Associative } \\
\text { Accumulation } \\
\text { Model (AAM) }\end{array}$ & Bhatia (2013) & $\mathrm{X}$ & & $\mathrm{X}$ & $\mathrm{X}$ & & \\
\hline $\begin{array}{l}\text { Multi-attribute } \\
\text { Linear Ballistic } \\
\text { Accumulator } \\
\text { (MLBA) }\end{array}$ & $\begin{array}{l}\text { Trueblood et al. } \\
\text { (2014) }\end{array}$ & $\mathrm{X}$ & $\mathrm{X}$ & & & & \\
\hline $\begin{array}{l}\text { Multi-alternative } \\
\text { Decision by } \\
\text { Sampling (MDbS) }\end{array}$ & $\begin{array}{l}\text { Noguchi \& } \\
\text { Stewart (2018) }\end{array}$ & $\mathrm{X}$ & $\mathrm{X}$ & & & & \\
\hline $\begin{array}{l}\text { Normalization and } \\
\text { Urgency Model }\end{array}$ & $\begin{array}{l}\text { Tajima et al. } \\
\text { (2019) }\end{array}$ & & & & & $\mathrm{X}$ & $\mathrm{X}$ \\
\hline $\begin{array}{l}\text { Selective Integration } \\
\text { Model }\end{array}$ & $\begin{array}{l}\text { Usher et al. } \\
\text { (2019) }\end{array}$ & $\mathrm{X}$ & $X$ & $\mathrm{X}$ & & & \\
\hline $\begin{array}{l}\text { Gaze-dependent } \\
\text { Leaky Accumulator } \\
\text { (GLA) }\end{array}$ & $\begin{array}{l}\text { Molter et al. } \\
(2021)\end{array}$ & $\mathrm{X}$ & & $X$ & & & \\
\hline
\end{tabular}

Note. Leakage accounts for the degradation and loss of information during the time course of the decision, i.e. the overweighting of new information relative to old. Urgency captures an individual's desire to reach a decision in a reasonable amount of time.

In Table 1, we list several different mechanisms that have been included in these models over the years. This list of mechanisms is not exhaustive, but includes the most important mechanisms discussed with respect to this set of models. To illustrate how various mechanisms 
can give rise to context effects, consider MDFT. In this model, two key mechanisms are needed to explain context effects: inhibition and attention. Inhibition is the capacity of one option to reduce the evidence accumulated for other options. Specifically, the attraction effect arises due to "negative inhibition" from the dominated decoy to the dominating option. Conceptually, this is akin to a double negative, leading to a boost in evidence accumulation for the dominating option. The similarity effect arises through attention switching between the two attributes. In the model, attention is either allocated to one attribute or the other at any moment in time. When attention is allocated to the attribute favoring the dissimilar alternative (e.g., the attribute "convenience" favoring option $\mathrm{X}$ in Figure 1), it gains an advantage. Likewise, when attention is allocated to the attribute favoring the similar alternatives (e.g., the attribute "economy" favoring Y and S in Figure 1), they gain an advantage. This dynamic attention switching process leads to a positive correlation in evidence accumulation between the similar options. Someone focusing their attention more on "convenience" will end up selecting option X. However, someone focusing their attention more on "economy" will select either Y or S, roughly equally due to the correlation in evidence accumulation. Like the attraction effect, the compromise effect arises through inhibition. In this case, negative inhibition results in evidence accumulation for the compromise option being negatively correlated with evidence for both extremes. This correlation leads to a probabilistic advantage for the compromise option due to the stochastic nature of the model.

Looking at Table 1, we see that models typically involve multiple mechanisms. As described above, attention and inhibition are critical to explaining context effects in MDFT. While different models evoke different mechanisms, there is one interesting similarity across almost all models. Eight out of nine models have attention mechanisms, suggesting attention is 
important for context effects. While the specific implementation of attention varies from model to model, the basic idea is the same. When someone is faced with multiple options that vary on multiple attributes, they cannot allocate attention to everything simultaneously. Rather, they allocate attention to a subset of information and accumulate evidence on the currently attended information. Throughout deliberation, individuals shift this "window of attention" to evaluate the various options. In MDFT, the window of attention includes all options along a single attribute. In MDbS (Noguchi \& Stewart, 2018), the window of attention includes a pair of options along a single attribute. In MLBA (Trueblood, Brown, \& Heathcote, 2014), the window of attention includes pairwise comparisons similar to MDbS; however, this is modeled through weight parameters rather than explicit shifts of attention due to the ballistic nature of evidence accumulation. In Table 2, we describe the window of attention (i.e., what is attended) and how attention is implemented in the eight models with attention mechanisms. As can be seen from the table, there are a variety of different ways attention has been interpreted in these models.

We note that one model, the Normalization and Urgency Model (Tajima, Drugowitsch, Patel, \& Pouget, 2019), does not include an attention mechanism. Rather this model accounts for context effects through divisive normalization (i.e., the phenomenon where the firing rate of a neuron is normalized by summing the firing rates of a pool of neurons and dividing by that sum). However, recent eye-tracking experiments suggest that value-based attention rather than divisive normalization influences multi-alternative choice behavior (Gluth, Kern, Kortmann, \& Vitali, 2020). While divisive normalization is thought to occur throughout the brain, more work is needed to understand if this neural computation has behavioral impacts that may lead to context effects (also see Landry \& Webb, 2021, for another model of context effects based on divisive normalization). 
Table 2

Attention Mechanisms in Dynamic Models of Context Effects

\begin{tabular}{|c|c|c|c|}
\hline Model Name & Reference & Window Attention & Implementation \\
\hline $\begin{array}{l}\text { Multi-alternative } \\
\text { Decision Field } \\
\text { Theory (MDFT) }\end{array}$ & $\begin{array}{l}\text { Roe et al. } \\
(2001)\end{array}$ & All options on one attribute & $\begin{array}{l}\text { Sequential focus on different attributes } \\
\text { over time where the attribute is selected } \\
\text { probabilistically }\end{array}$ \\
\hline $\begin{array}{l}\text { Leaky Competing } \\
\text { Accumulator (LCA) }\end{array}$ & $\begin{array}{l}\text { Usher \& } \\
\text { McClelland } \\
\text { (2004) }\end{array}$ & All options on one attribute & $\begin{array}{l}\text { Sequential focus on different attributes } \\
\text { over time where the attribute is selected } \\
\text { probabilistically }\end{array}$ \\
\hline 2N-ary Choice Tree & $\begin{array}{l}\text { Wollschlager \& } \\
\text { Diederich } \\
\text { (2012) }\end{array}$ & One option on one attribute & $\begin{array}{l}\text { Sequential focus on single options and } \\
\text { attributes over time where the option and } \\
\text { attribute are selected probabilistically }\end{array}$ \\
\hline $\begin{array}{l}\text { Associative } \\
\text { Accumulation } \\
\text { Model (AAM) }\end{array}$ & Bhatia (2013) & All options on one attribute & $\begin{array}{l}\text { Sequential focus on different attributes } \\
\text { over time where the attribute is selected } \\
\text { probabilistically }\end{array}$ \\
\hline $\begin{array}{l}\text { Multi-attribute } \\
\text { Linear Ballistic } \\
\text { Accumulator } \\
\text { (MLBA) }\end{array}$ & $\begin{array}{l}\text { Trueblood et al. } \\
\text { (2014) }\end{array}$ & $\begin{array}{l}\text { Pairs of options on one } \\
\text { attribute }\end{array}$ & $\begin{array}{l}\text { Weights on pairwise comparisons where } \\
\text { the magnitude of the weight is } \\
\text { determined by the similarity of the pair } \\
\text { of options on one attribute }\end{array}$ \\
\hline $\begin{array}{l}\text { Multi-alternative } \\
\text { Decision by } \\
\text { Sampling (MDbS) }\end{array}$ & $\begin{array}{l}\text { Noguchi \& } \\
\text { Stewart (2018) }\end{array}$ & $\begin{array}{l}\text { Pairs of options on one } \\
\text { attribute }\end{array}$ & $\begin{array}{l}\text { Sequential focus on a pair of options and } \\
\text { one attribute over time selected } \\
\text { probabilistically }\end{array}$ \\
\hline $\begin{array}{l}\text { Selective Integration } \\
\text { Model }\end{array}$ & $\begin{array}{l}\text { Usher et al. } \\
(2019)\end{array}$ & $\begin{array}{l}\text { A sample from all options in } \\
\text { the choice set }{ }^{\mathrm{a}}\end{array}$ & $\begin{array}{l}\text { Goal-dependent attentional weights on } \\
\text { sampled values }\end{array}$ \\
\hline $\begin{array}{l}\text { Gaze-dependent } \\
\text { Leaky Accumulator } \\
\text { (GLA) }\end{array}$ & $\begin{array}{l}\text { Molter et al. } \\
(2021)\end{array}$ & One option & $\begin{array}{l}\text { Sequential focus on different options } \\
\text { based on fixations using eye-tracking }\end{array}$ \\
\hline
\end{tabular}

While attention is common to almost all models of context effects, other mechanisms in Table 1 appear in only some of the models. For example, several theories incorporate the relative evaluation of options. In these models, the evaluation of an option occurs by comparing the option to an internal reference point (i.e., a reference point determined by the other options in the choice set). In MDFT, an option is evaluated by comparing it to the mean of the other options. In 
other models such as the LCA (Usher, \& McClelland, 2004), MDbS, and MLBA, an option is evaluated through pairwise comparisons along a single attribute. The idea of pairwise comparisons originates from Tversky \& Simonson's (1993) early modeling work on context effects. While several models explicitly incorporate relative comparisons, others do not. Thus, it remains an open question of how important relative comparisons are to context effects.

The past two decades have seen the development of many different models of context effects. As discussed in this section, these models have similarities and differences (see also Busemeyer, Gluth, Rieskamp, \& Turner, 2019, for review). While traditional model comparison focuses on evaluating the differences among models, there is also utility in considering the similarities. In particular, we see that most theories of multi-alternative, multi-attribute choice incorporate attention mechanisms, although how attention is implemented varies across models as illustrated in Table 2. This suggests that future research should more carefully study the relationship between attention and context effects in order to refine our understanding of this critical mechanism. Below we describe some recent empirical work along these lines.

\section{Empirical Studies of Attention and Context Effects}

Recent research on context effects has examined the impact of attentional and comparative processes by (1) measuring visual attention using eye-tracking and (2) manipulating the layout of options on the screen. With respect to the former, one of the first studies to use eyetracking to understand gaze patterns in context effects was Noguchi and Stewart (2014). They found that eye-movements revealed a series of comparisons between pairs of alternatives along a single dimension consistent with many dynamic models of context effects, including MDbS, MLBA, the 2N-ary Choice Tree (see Table 2). More recently, Marini, Ansani, \& Paglieri (2020) used eye-tracking to understand how dominated decoys impact eye-movements in the attraction 
effect. They found that decoys result in more consecutive shifts of attention between the decoy and target, suggesting an increase in comparing target and decoy options during choice. Molter et al. (2021) found that target options received greater relative dwell time than competitor options in both the attraction and compromise effects. Overall, these results show that attention and how it shifts during deliberation plays a key role in understanding context effects.

Other studies have revealed the impact of presentation layout on the strength of context effects, shedding light on how attention could modulate the effects. For example, Evans et al. (2021) reanalyzed data from Trueblood, Brown, Heathcote, \& Busemeyer (2013) and found that the spatial ordering (i.e., right to left placement of alternatives on the screen) impacted the strength of the attraction effect. In orderings where the target and decoy were adjacent, a standard effect was generally observed. However, when the competitor came between the target and decoy (i.e., it was the middle option), a reversed attraction effect occurred. These results likely arise because it is easier to shift attention between (and thus compare) adjacent options. When the competitor is the middle option, it potentially receives more attention and thus accumulates evidence (preference) faster than the other options, leading to a reversal of the attraction effect. He and Sternthal (2018) found a similar effect of spatial order on the attraction effect in consumer choice tasks. Likewise, Spektor et al. (2018) found strong reversals of the attraction effect when options were spatially arranged far apart in a triangular configuration.

Related research by Cataldo \& Cohen (2019) found that manipulating the presentation format of a choice set (i.e., the arrangement of options in a table) impacted the presence of context effects. They found standard attraction and compromise effects, but a reversed similarity effect in a "by-dimension" format (i.e., options are displayed in separate rows with their attributes listed in different columns). However, they found a null attraction effect and reversed 
compromise effect, but a standard similarity effect in a "by-alternative" format (i.e., options are displayed in separate columns with their attributes listed in different rows). Similar to the findings discussed above on spatial arrangement, these results likely arise from the interaction of attention and choice set layout.

Attention not only influences what individuals look at, but also how they encode information into memory. The effects of attention on memory are also relevant when thinking about context effects. For example, Bhatia (2014) found that participants had increased memory for features shared by target and decoy options (as measured using a recall task), which mediated the strength of the attraction effect. In this case, attention to the shared features of the target and decoy likely enhanced the encoding of these features in memory, which then impacted behavior.

\section{Discussion}

For decades, context effects have been a primary focus of investigations into multialternative, multi-attribute decision-making. Originally studied to demonstrate violations of classic decision theories, in recent years, context effects have helped researchers investigate the cognitive and neural mechanisms underlying multi-alternative, multi-attribute choice. As discussed in this review, there are many different theoretical accounts of context effects. While attention is common to most dynamic models of context effects, divisive normalization offers an alternative explanation. It is possible that there are multiple pathways to context effects. Such a view might explain why context effects often appear ubiquitous, occurring in different domains (Trueblood et al., 2013) and species (Latty \& Trueblood, 2020).

When thinking about empirical and theoretical developments with regards to context effects, an important outstanding problem is understanding reversals of these effects. Recent studies have documented reversals of context effects (see Spektor, Bhatia, \& Gluth, 2021, for a 
review), but it is unknown when and why reversals occur. On the one hand, this has caused some researchers to question the importance of these effects (Frederick, Lee, \& Baskin, 2014). On the other hand, this provides researchers an opportunity to refine our understanding of how fundamental cognitive processes, such as attention, play a role in multi-alternative, multiattribute choice. It will require both new empirical and computational modeling work to elucidate these processes.

On the empirical side, new experiments are needed to explore the factors that lead to reversals. We hypothesize that attention might be critical to understanding reversals based on the research showing that presentation layout influences the effects. However, it still remains unclear exactly how presentation layout impacts attention. Eye-tracking studies that manipulate layout could help shed light on this question.

On the theoretical side, research is needed to understand the cognitive mechanisms responsible for context effect reversals. Many of the existing models discussed in this review cannot fully account for recent empirical demonstrations of context effect reversals or how choices change over time (Cataldo \& Cohen, 2021). Thus, new modeling approaches are needed. The "switchboard" modeling approach of Turner et al. (2018) could prove very useful in exploring the space of models that can produce both standard and reversed context effects along with identifying the relevant underlying cognitive mechanisms. Importantly, this approach could help distinguish among the various implementations of attention listed in Table 2. Another important goal for future research will be using information about how choices change over time to evaluate models. To date, there have only been a few studies evaluating models using both choices and response times (e.g., Evans et al., 2019; Cataldo \& Cohen, 2021). In sum, coupling the new modeling approaches and empirical studies aimed at manipulating the presence / 
absence of context effects could reveal new insights into the dynamics of multi-alternative, multi-attribute decision-making and how preferences evolve over time. 


\section{Recommended Reading}

Busemeyer, J. R., Gluth, S., Rieskamp, J., \& Turner, B. M. (2019). (See References). A review of computational and neuroscience research on multi-alternative value-based decision-making.

Evans, N. J., Holmes, W. R., \& Trueblood, J. S. (2019). (See References). A quantitative comparison of four dynamic models of context effects using both choice and response time data.

Spektor, M. S., Bhatia, S., \& Gluth, S. (2021). (See References). A review of empirical studies showing reversals in context effects and discussing potential boundary conditions of these effects.

Turner, B. M., Schley, D. R., Muller, C., \& Tsetsos, K. (2018). (See References). A quantitative comparison of dynamic models of context effects using the switchboard analysis approach applied to choice data. 
Acknowledgments

This work was supported by NSF grant 1846764 . 


\section{References}

Bhatia, S. (2013). Associations and the accumulation of preference. Psychological review, 120(3), 522.

Bhatia, S. (2014). Confirmatory search and asymmetric dominance. Journal of Behavioral Decision Making, 27(5), 468-476.

Busemeyer, J. R., Gluth, S., Rieskamp, J., \& Turner, B. M. (2019). Cognitive and neural bases of multi-attribute, multi-alternative, value-based decisions. Trends in cognitive sciences, 23(3), 251263.

Cataldo, A. M., \& Cohen, A. L. (2019). The comparison process as an account of variation in the attraction, compromise, and similarity effects. Psychonomic Bulletin \& Review, 26(3), 934-942.

Cataldo, A. M., \& Cohen, A. L. (2021). Modeling preference reversals in context effects over time. Computational Brain \& Behavior, 4(1), 101-123.

Dumbalska, T., Li, V., Tsetsos, K., \& Summerfield, C. (2020). A map of decoy influence in human multialternative choice. Proceedings of the National Academy of Sciences, 117(40), 25169-25178.

Evans, N. J., Holmes, W. R., Dasari, A., \& Trueblood, J. S. (2021). The impact of presentation order on attraction and repulsion effects in decision-making. Decision, 8(1), 36.

Evans, N. J., Holmes, W. R., \& Trueblood, J. S. (2019). Response-time data provide critical constraints on dynamic models of multi-alternative, multi-attribute choice. Psychonomic Bulletin \& Review, 26(3), 901-933.

Frederick, S., Lee, L., \& Baskin, E. (2014). The limits of attraction. Journal of Marketing Research, 51(4), 487-507.

Gluth, S., Kern, N., Kortmann, M., \& Vitali, C. L. (2020). Value-based attention but not divisive normalization influences decisions with multiple alternatives. Nature Human Behaviour, 4(6), 634-645.

He, S., \& Sternthal, B. (2018). Explaining the attraction effect: an ambiguity-attentionapplicability framework. ACR North American Advances.

Huber, J., Payne, J. W., \& Puto, C. (1982). Adding asymmetrically dominated alternatives: Violations of regularity and the similarity hypothesis. Journal of Consumer Research, 9, 90-98. doi:10.1086/208899

Latty, T., \& Trueblood, J. S. (2020). How do insects choose flowers? A review of multi-attribute flower choice and decoy effects in flower-visiting insects. Journal of Animal Ecology, 89(12), 2750-2762. 
Luce, R. D. (1959). Individual choice behavior: A theoretical analysis. New York, NY: Wiley.

Marini, M., Ansani, A., \& Paglieri, F. (2020). Attraction comes from many sources: Attentional and comparative processes in decoy effects. Judgment \& Decision Making, 15(5).

Molter, F., Thomas, A., Huettel, S. A., Heekeren, H., \& Mohr, P. N. C. (2021, February 12). Gaze-dependent evidence accumulation predicts multi-alternative risky choice behaviour. https://doi.org/10.31234/osf.io/x6nbf

Noguchi, T., \& Stewart, N. (2014). In the attraction, compromise, and similarity effects, alternatives are repeatedly compared in pairs on single dimensions. Cognition, 132(1), 44-56.

Noguchi, T., \& Stewart, N. (2018). Multialternative decision by sampling: A model of decision making constrained by process data. Psychological review, 125(4), 512.

Pettibone, J. C. (2012). Testing the effect of time pressure on asymmetric dominance and compromise decoys in choice. Judgment and Decision making, 7(4), 513.

Rigoli, F., Mathys, C., Friston, K. J., \& Dolan, R. J. (2017). A unifying Bayesian account of contextual effects in value-based choice. PLoS computational biology, 13(10), e1005769.

Simonson, I. (1989). Choice based on reasons: The case of attraction and compromise effects. Journal of Consumer Research, 16, 158-174.

Spektor, M. S., Bhatia, S., \& Gluth, S. (2021). The elusiveness of context effects in decision making. Trends in Cognitive Sciences.

Spektor, M. S., Kellen, D., \& Hotaling, J. M. (2018). When the good looks bad: An experimental exploration of the repulsion effect. Psychological science, 29(8), 1309-1320.

Ratcliff, R. (1978). A theory of memory retrieval. Psychological Review, 85, 59-108. doi:10.1037/0033-295X.85.2.59

Roe, R. M., Busemeyer, J. R., \& Townsend, J. T. (2001). Multialternative decision field theory: A dynamic connectionist model of decision making. Psychological Review, 108, 370-392. doi:10.1037/0033-295X.108.2.370

Tajima, S., Drugowitsch, J., Patel, N., \& Pouget, A. (2019). Optimal policy for multi-alternative decisions. Nature neuroscience, 22(9), 1503-1511.

Trueblood, J. S., Brown, S. D., \& Heathcote, A. (2014). The multiattribute linear ballistic accumulator model of context effects in multialternative choice. Psychological review, 121(2), 179. 
Trueblood, J. S., Brown, S. D., Heathcote, A., \& Busemeyer, J. R. (2013). Not just for consumers: Context effects are fundamental to decision making. Psychological science, 24(6), 901-908.

Turner, B. M., Schley, D. R., Muller, C., \& Tsetsos, K. (2018). Competing theories of multialternative, multiattribute preferential choice. Psychological review, 125(3), 329.

Tversky, A. (1972). Elimination by aspects: A theory of choice. Psychological review, 79(4), 281.

Tversky, A., \& Simonson, I. (1993). Context dependent preferences. Management Science, 39, 1179-1189. doi:10.1287/mnsc.39.10.1179

Usher, M., \& McClelland, J. L. (2004). Loss aversion and inhibition in dynamical models of multialternative choice. Psychological Review, 111, 757-769. doi:10.1037/0033-295X.111.3.757

Usher, M., Tsetsos, K., Glickman, M., \& Chater, N. (2019). Selective integration: an attentional theory of choice biases and adaptive choice. Current Directions in Psychological Science, 28(6), $552-559$.

Landry, P., \& Webb, R. (2021). Pairwise normalization: A neuroeconomic theory of multiattribute choice. Journal of Economic Theory, 193, 105221.

Wollschläger, L. M., \& Diederich, A. (2012). The 2N-ary choice tree model for N-alternative preferential choice. Frontiers in Psychology, 3, 189. 